\title{
KINETICS OF STYRENE SLURRY POLYMERIZATION IN SUSPENDED ISOOCTANE DROPLETS
}

\author{
YASUO HATATE, YOUICHI KUROKAWA, HIROSHI HAMADA, \\ ATSUSHI IKARI AND FUMIYUKI NAKASHIO \\ Department of Chemical Engineering, Kagoshima University, Kagoshima 890
}

Key Words: Kinetics, Styrene, Slurry Polymerization, Average Molecular Weight, Microcapsule, Twophase Separation Model

\begin{abstract}
Styrene slurry polymerization in isooctane droplets suspended in water was carried out to clarify the characteristics. The following results were obtained: (1) revolution rate, existence of PVA and volume fraction of dispersion phase have no effect on the kinetics of the polymerization, and (2) the effect of initiator concentration on the kinetics in this polymerization is similar to that in common homopolymerization, while the effect of monomer concentration is different from that in homopolymerization. At low monomer concentrations in particular, the polymers produced have much higher molecular weight than those in homopolymerization.

A two-phase separation model is proposed to describe the characteristics of the polymerization, and the kinetic equations are derived. The kinetic parameters were determined to show comparatively good agreement between calculated and observed relations of conversion and average molecular weight with reaction time.
\end{abstract}

\section{Introduction}

The adherence to the reactor wall and the agglomeration of viscous polymer droplets in suspension polymerization make its operating applicability limited. In slurry polymerization the adherence problem is also the most important one to be solved in designing the reactor. ${ }^{7)} \mathrm{A}$ slurry polymerization in suspended droplets in water was proposed as a useful method to reduce such a problem, and experiments using styrene as a monomer were carried out to confirm the drastic reduction of adherence to the reactor wall. Furthermore, it was found that microparticles, consisting of solvent within and polymer as the outside shell, are formed due to in situ coagulation. Applicability to a microcapsule production method was suggested. However, the kinetics of this polymerization is hardly known so far.

In the present study, styrene slurry polymerization in suspended isooctane droplets was carried out under a wide range of operating conditions to clarify the kinetic characteristics, and a new theoretical model was developed after the treatment of Hamielec et al. of vinyl chloride slurry polymerization. ${ }^{1}$

\section{Polymerization Kinetic Equations}

Elementary reactions in homogeneous radical solution polymerization are generally represented in Table 1. Monomer consumption rate and production rate of dead polymer having $r$ monomer units are Received September 13, 1984. Correspondence concerning this article should be
addressed to Y. Hatate. F. Nakashio is at Dept. of Org. Synth., Kyushu Univ., Fukuoka 812. respectively shown by Eqs. (1) and (2) as shown previously for solution copolymerization. ${ }^{5}$

$$
\begin{gathered}
R_{p}=-\rho \frac{d(A / \rho)}{d t}=\frac{k_{p}}{k_{t}^{1 / 2}} \frac{R_{i}^{1 / 2} A}{\left(1+2 H_{1} T_{f S} S / A\right)^{1 / 2}} \\
\rho \frac{d\left(M_{r} / \rho\right)}{d t}=\zeta^{r}(1-\zeta) R_{p}\left[T_{f A}+T_{f S} \frac{S}{A}+\frac{R_{i}}{R_{p}}\right. \\
\left.\times \frac{\left\{k_{t d}+0.5 k_{t c}(r-1)(1-\zeta)\right\} / k_{t}+H_{1} T_{f S} S / A}{1+2 H_{1} T_{f S} S / A}\right]
\end{gathered}
$$

where

$$
\begin{aligned}
& R_{i}=2 k_{d} f I, \quad H_{1}=\left(k_{t S} / k_{t}\right) /\left(k_{i S} / k_{p}\right) \\
& \zeta=1-P_{1} / \sum_{r=1}^{\infty} P_{r}
\end{aligned}
$$

Instantaneous number and weight average degrees of polymerization are respectively shown by Eqs. (3) and (4).

Table 1. Kinetic scheme

$\begin{array}{cc}\text { Initiation } & \text { Kinetic rate constant } \\ I & k_{d} \\ R+A \longrightarrow 2 R & k_{i} \\ \text { Propagation } & \\ P_{r}+A \longrightarrow P_{l} & k_{P} \\ \text { Chain transfer } & \\ P_{r}+A \longrightarrow P_{r+1} \longrightarrow M_{r}+P_{1} & k_{f A} \\ P_{r}+S \longrightarrow M_{r}+S^{*} & k_{f S} \\ \text { Reinitiation by solvent radical } & \\ S^{*}+A \longrightarrow P_{1} & k_{i S} \\ \text { Termination } & \\ P_{r}+P_{s} \longrightarrow M_{r+s} & k_{i c} \\ P_{r}+P_{s} \longrightarrow M_{r}+M_{s} & k_{t d} \\ \text { Termination by solvent radical } & k_{t s} \\ P_{r}+S^{*} \longrightarrow M_{r} & \end{array}$




$$
\begin{gathered}
\bar{P}_{N, \text { inst }}=\sum_{r=1}^{\infty} r\left(d M_{r} / d t\right) / \sum_{r=1}^{\infty}\left(d M_{r} / d t\right) \\
=\left(T_{f A}+T_{f S} \frac{S}{A}+\frac{R_{i}}{2 R_{p}} \frac{\left(2 k_{t d}+k_{t c}\right) / k_{t}+2 H_{1} T_{f S} S / A}{1+2 H_{1} T_{f S} S / A}\right)^{-1} \\
\bar{P}_{W, \text { inst }}=\sum_{r=1}^{\infty} r^{2}\left(d M_{r} / d t\right) / \sum_{r=1}^{\infty} r\left(d M_{r} / d t\right) \\
=2 \bar{P}_{N, \text { inst }} \frac{\left(1+\frac{R_{i}}{R_{p}} \frac{\bar{P}_{N, \text { inst }} k_{c c} / k_{t}}{1+2 H_{1} T_{f S} S / A}\right)}{\left(1+\frac{R_{i}}{2 R_{p}} \frac{\bar{P}_{N, \text { inst }} k_{t c} / k_{t}}{1+2 H_{1} T_{f S} S / A}\right)^{2}}
\end{gathered}
$$

\section{Experimental}

\subsection{Reagent}

Styrene and $\alpha, \alpha^{\prime}$-azobisisobutylonitrile (AIBN) were purified by the same method as mentioned previously. ${ }^{4}$ Ion-exchanged water was distilled under reduced pressure of nitrogen before use. $0.1 \mathrm{wt} \%$ polyvinyl alcohol (PVA) aqueous solution was prepared by feeding a given amount of water with a given amount of PVA (degree of polymerization $=1500$ ) in an atmosphere of nitrogen. Isooctane was purified by distillation under reduced pressure of nitrogen.

\subsection{Experimental apparatus and procedure}

A schematic diagram of the experimental apparatus is shown in Fig. 1. The reactor was a $1000-\mathrm{m} l$ separable flask with four baffle blades having a width of $1 / 10$ the reactor diameter. A two-bladed paddle was used for mixing, as shown in Fig. 1.

The experimental procedure is described below. First, after air in the reactor system was replaced with nitrogen, desired amounts of water, isooctane, styrene and initiator were stored in each burette and a container for initiator in an atmosphere of nitrogen. Secondly, prescribed amounts of water and isooctane were charged into the reactor and the temperature in the reactor was left to reach $70^{\circ} \mathrm{C}$ at a given revolution rate. Finally, the initiator was dissolved in a prescribed amount of styrene and was rapidly introduced into the reactor using nitrogen pressure.*1 From this time samples were taken at regular intervals from the three-way cock by charging the reactor with nitrogen. The weights of both polymer and monomer present in each sample were determined by the precipitation method and steam gas chromatography. Monomer conversion was calculated from the following equation.

$$
\text { conversion }=\{\text { polymer weight }(\mathrm{g})\} /\{\text { monomer }
$$

$$
\text { weight }(\mathrm{g})+\text { polymer weight }(\mathrm{g})\}
$$

${ }^{* 1}$ No coalescence effect on the mixing of styrene and isooctane is expected ${ }^{6}{ }^{6}$ In this study, this was confirmed by carrying out an experiment with a premixed feed of styrene and isooctane.

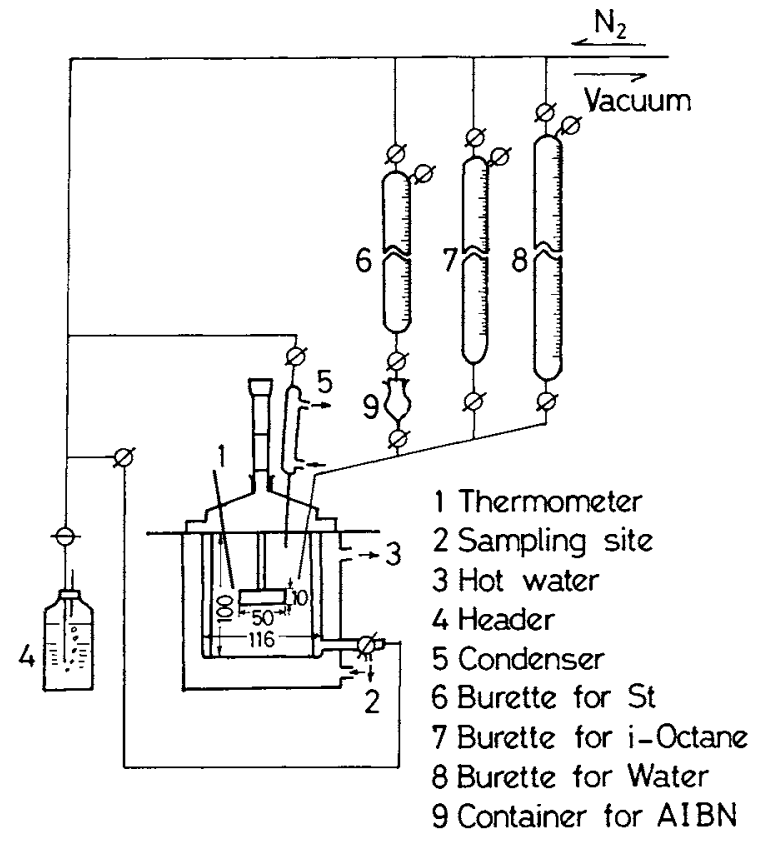

Fig. 1. Experimental apparatus.

The molecular weight distribution of the polymer obtained above was determined by high-speed liquid chromatography.

\section{Result and Discussion}

\subsection{Effect of operating conditions on polymerization}

The experimental conditions are shown in Table 2. In this work the following operating conditions were examined; 1) revolution rate, 2) existence of dispersion stabilizer PVA, 3) dispersion phase volume fraction, 4) initiator concentration and 5) monomer concentration.

3.1.1 Effect of revolution rate, PVA and dispersion phase fraction on polymerization To demonstrate the effect of revolution rate, plots of monomer conversion $(x)$ and average molecular weight $(\bar{M})$ vs. reaction time $(t)$ are respectively represented in Figs. 2 and 3 , covering the range of 650 to $1400 \mathrm{rpm}$. It is evident from these figures that no effect of revolution rate on polymerization kinetics is detectable.

Solid lines in the figures represent calculated results from the model described in section 3.2. The same is true for figures on time dependencies of conversion and molecular weight.

Plots of $x$ and $\bar{M}$ vs. $t$ are respectively represented in Figs. 4 and 5, where $0.1 \mathrm{wt} \%$ PVA and no. PVA are used for comparison. No effect of PVA on polymerization kinetics is evident from these figures.

Plots of $x$ and $\bar{M}$ vs. $t$ are respectively represented in Figs. 6 and 7, where 0.2 and 0.4 of initial disersionphase volume fraction $\left(\psi_{0}\right)$ are used for comparison. Almost no effect of dispersion-phase fraction on the polymerization kinetics is also evident over a wide 
Table 2. Experimental conditions

\begin{tabular}{|c|c|c|c|c|c|c|}
\hline Run No. & $\begin{array}{c}A_{0} \\
{[\mathrm{~mol} / \mathrm{l}]}\end{array}$ & $\begin{array}{c}I_{0} \\
{[\mathrm{~mol} / \mathrm{l}]}\end{array}$ & $\begin{array}{c}\psi_{0} \\
{[-]}\end{array}$ & $\begin{array}{l}\text { Rev. rate } \\
{\left[\min ^{-1}\right]}\end{array}$ & $\begin{array}{c}\text { PVA } \\
{[w t \%]}\end{array}$ & Key \\
\hline 202 & 8.30 & 0.010 & 0.20 & 650 & 0.1 & \\
\hline 203 & 8.30 & 0.038 & 0.20 & 650 & 0.1 & $\theta$ \\
\hline 206 & 5.77 & 0.038 & 0.20 & 650 & 0.1 & 0 \\
\hline 12 & 5.31 & 0.049 & 0.17 & 650 & 0 & $\square$ \\
\hline 3 & 5.15 & 0.051 & 0.16 & 650 & 0.1 & $\boxminus$ \\
\hline 2 & 5.15 & 0.051 & 0.16 & 1000 & 0.1 & \\
\hline 6 & 5.14 & 0.206 & 0.16 & 650 & 0.1 & $\square$ \\
\hline 10 & 5.14 & 0.013 & 0.16 & 650 & 0.1 & U \\
\hline 9 & 5.13 & 0.051 & 0.038 & 650 & 0.1 & \\
\hline 11 & 5.13 & 0.0032 & 0.16 & 650 & 0.1 & \\
\hline 5 & 4.51 & 0.052 & 0.14 & 650 & 0.1 & $\triangle$ \\
\hline 8 & 4.50 & 0.057 & 0.14 & 650 & 0.1 & $\triangle$ \\
\hline 204 & 3.88 & 0.038 & 0.20 & 650 & 0.1 & $A$ \\
\hline 305 & 3.84 & 0.010 & 0.20 & 1000 & 0 & \\
\hline 306 & 3.84 & 0.010 & 0.20 & 1000 & 0.1 & 0 \\
\hline 109 & 3.83 & 0.011 & 0.20 & 1400 & 0.1 & \\
\hline 112 & 3.83 & 0.038 & 0.40 & 1000 & 0.1 & 0 \\
\hline 113 & 3.83 & 0.0024 & 0.20 & 1000 & 0.1 & $\diamond$ \\
\hline 115 & 3.83 & 0 & 0.20 & 1000 & 0.1 & \\
\hline 201 & 3.83 & 0.010 & 0.20 & 650 & 0.1 & \\
\hline 114 & 3.76 & 0.151 & 0.20 & 1000 & 0.1 & $\downarrow$ \\
\hline 4 & 3.01 & 0.060 & 0.14 & 650 & 0.1 & $\bullet$ \\
\hline 205 & 2.40 & 0.034 & 0.20 & 650 & 0.1 & $\nabla$ \\
\hline 14 & 1.92 & 0.0024 & 0.20 & 650 & 0.1 & $\nabla$ \\
\hline 111 & 1.92 & 0.038 & 0.20 & 1000 & 0.1 & $\nabla$ \\
\hline 13 & 1.90 & 0.009 & 0.20 & 650 & 0.1 & $\square$ \\
\hline 7 & 1.65 & 0.066 & 0.13 & 650 & 0.1 & $\square$ \\
\hline 110 & 0.96 & 0.038 & 0.20 & 1000 & 0.1 & $凶$ \\
\hline
\end{tabular}

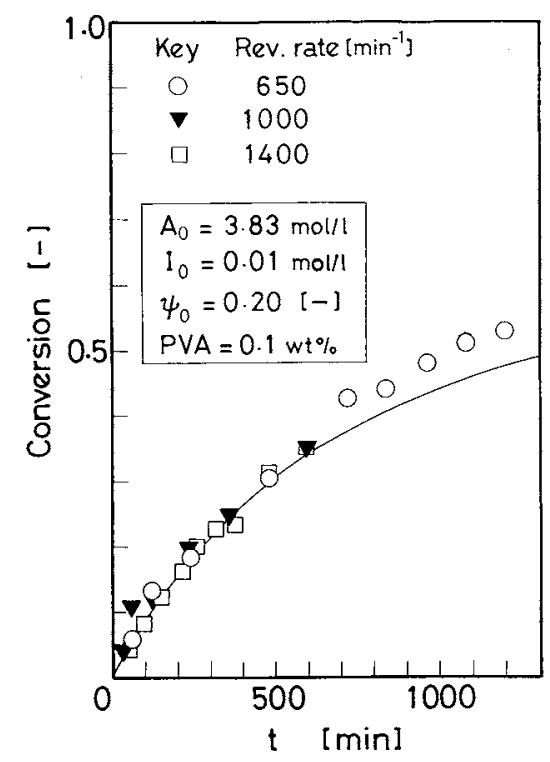

Fig. 2. Effect of revolution rate on polymerization rate.

range of the polymerization condition from these figures.

3.1.2 Effect of initiator concentration on polymerization To investigate the effect of initiator concentration at a comparatively high initial monomer concentration $A_{0}=5.1 \mathrm{~mol} / l$, plots of $x$ and $\bar{M}$ vs. $t$ are respectively shown in Figs. 8 and 9, covering the range of $I_{0}=0.003$ to $0.21 \mathrm{~mol} / l$. In these cases

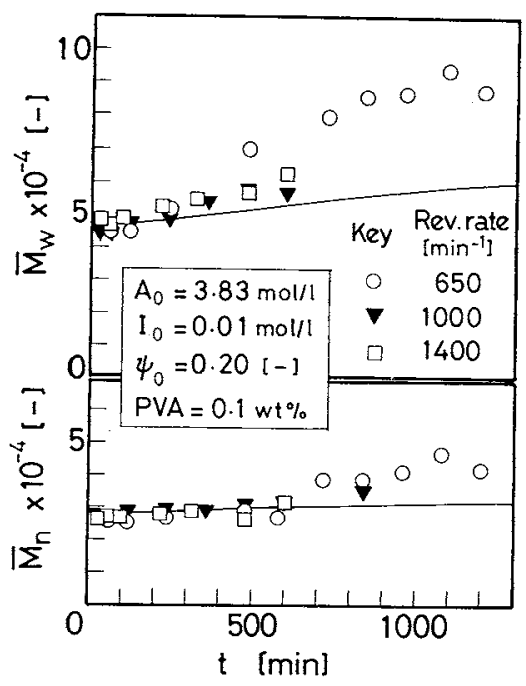

Fig. 3. Effect of revolution rate on average molecular weight.

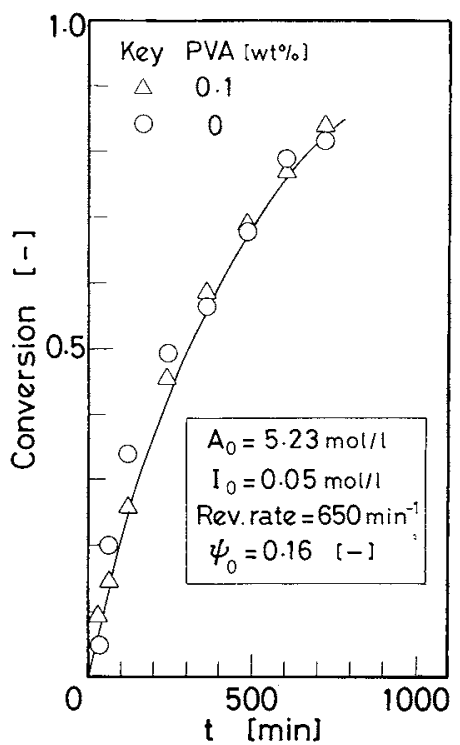

Fig. 4. Effect of PVA on polymerization rate.

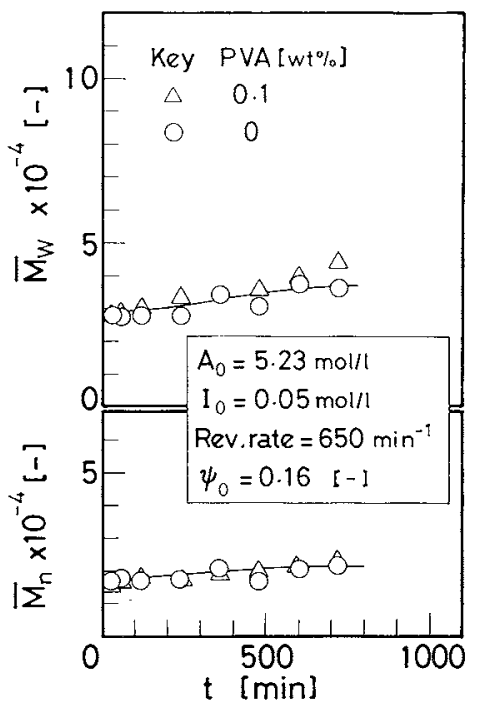

Fig. 5. Effect of PVA on average molecular weight. 


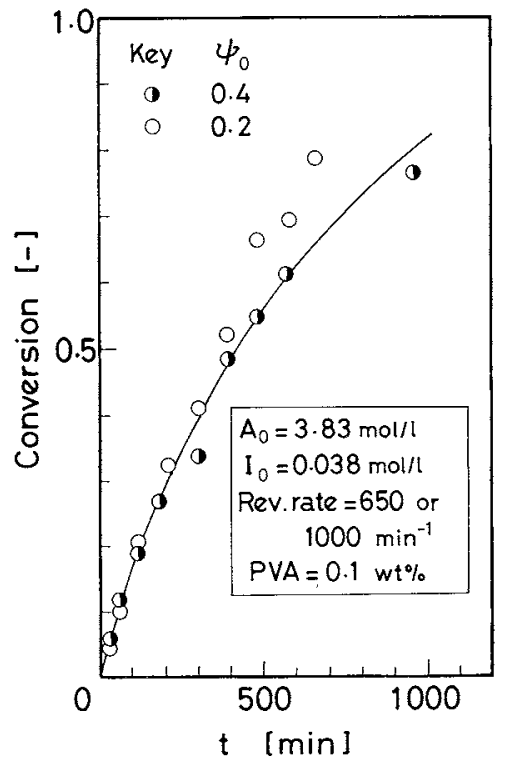

Fig. 6. Effect of dispersion-phase volume fraction on polymerization rate.

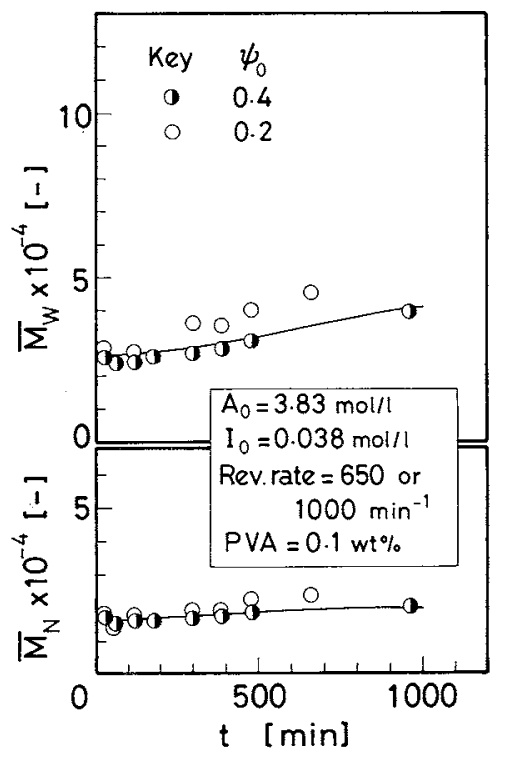

Fig. 7. Effect of dispersion-phase volume fraction on average molecular weight.

homogeneous polymerization is predominant at low monomer conversions. The same performance as for common homogeneous polymerization is recognized, at least at the initial stage of polymerization. The relation between initial polymerization rate $R_{P 0}$ and initial initiator concentration $I_{0}$ is shown in Fig. 10 . The common relation of $R_{P 0} \propto I_{0}^{1 / 2}$ in the radical polymerization is obvious in the figure. Since recombination termination is predominant in styrene polymerization, Eq. (3) is expressed by the following equation:

$$
\frac{1}{\bar{P}_{N}}=T_{f A}+T_{f S} \frac{S}{A}+\frac{R_{i}}{2 R_{p}}=T_{f A}+T_{f S} \frac{S}{A}+k_{d} f \frac{I}{R_{p}}
$$

A plot of $1 / \bar{P}_{N 0}$ vs. $I_{0} / R_{P 0}$ is shown in Fig. 11, and a

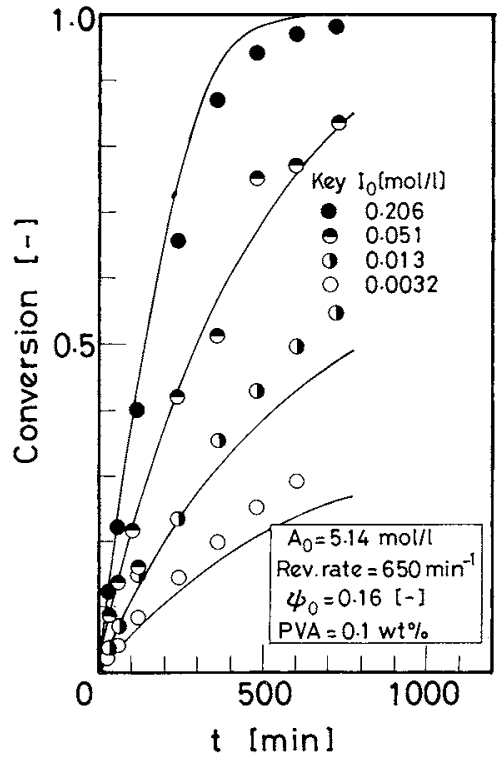

Fig. 8. Effect of initiator concentration on polymerization rate $\left(A_{0}=5.14 \mathrm{~mol} / \mathrm{l}\right)$.

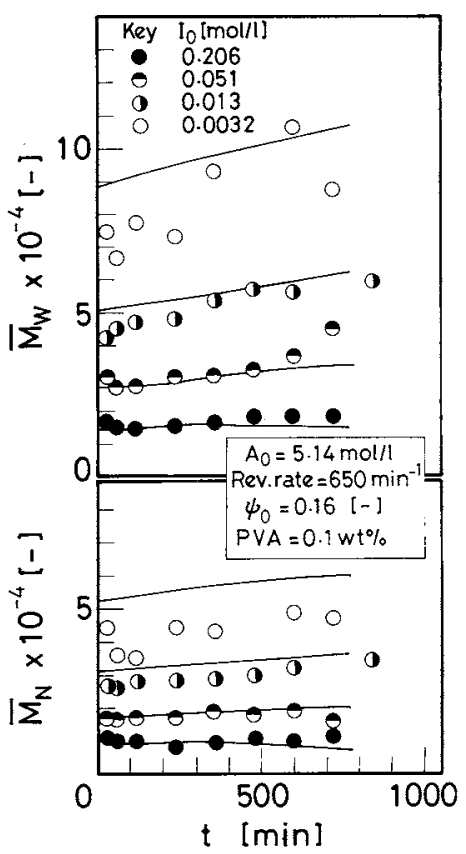

Fig. 9. Effect of initiator concentration on average molecular weight $\left(A_{0}=5.14 \mathrm{~mol} / \mathrm{l}\right)$.

linear relationship between $1 / \bar{P}_{N O}$ and $I_{0} / R_{P 0}$ is confirmed. Values of $\left(T_{f A}+T_{f S} S / A\right)$ and $k_{d} f$ are respectively obtained from the intersection at the ordinate axis and the slope of the linear line.

To investigate the effect of initiator concentration at a comparatively low initial monomer concentration $A_{0}=2 \mathrm{~mol} / l$, plots of $x$ and $\bar{M}$ vs. $t$ are respectively shown in Figs. 12 and 13. The initial rate of monomer conversion in polymerization exhibits almost the same tendency as in homogeneous polymerization. However, a linear relation with different slope is found between $1 / \bar{P}_{N 0}$ and $I_{0} / R_{P 0}$. If a constant value 


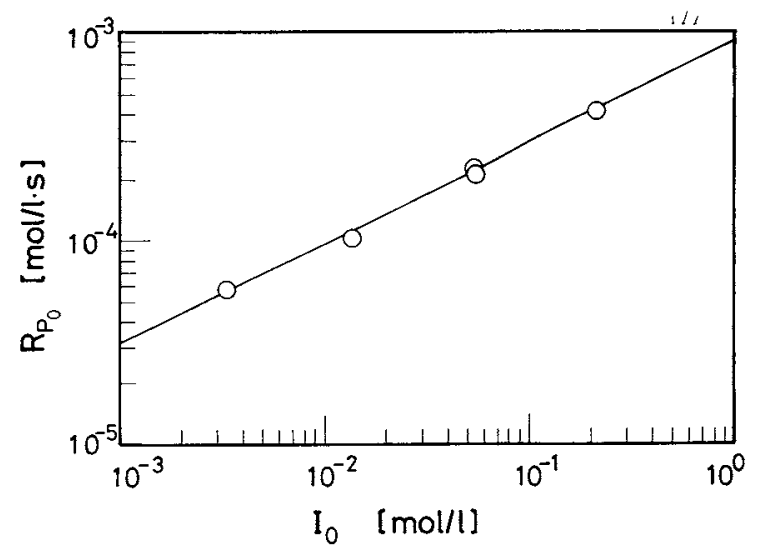

Fig. 10. Relation between $R_{P_{0}}$ and $I_{0}$.

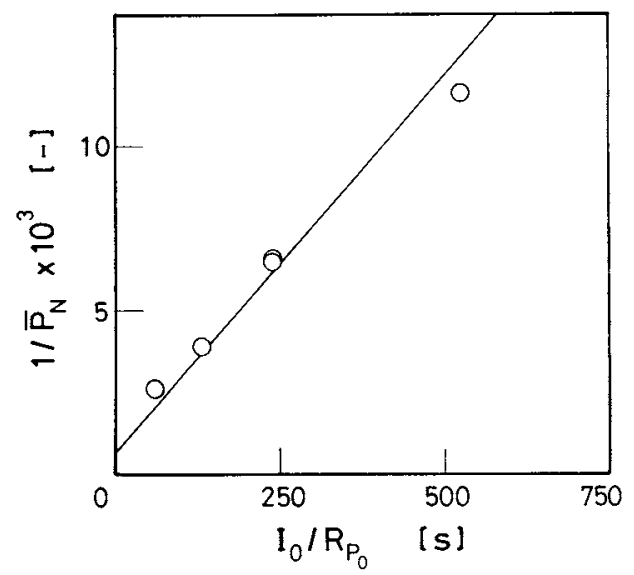

Fig. 11. Relation between $1 / \bar{P}_{N O}$ and $I_{0} / R_{P 0}$.

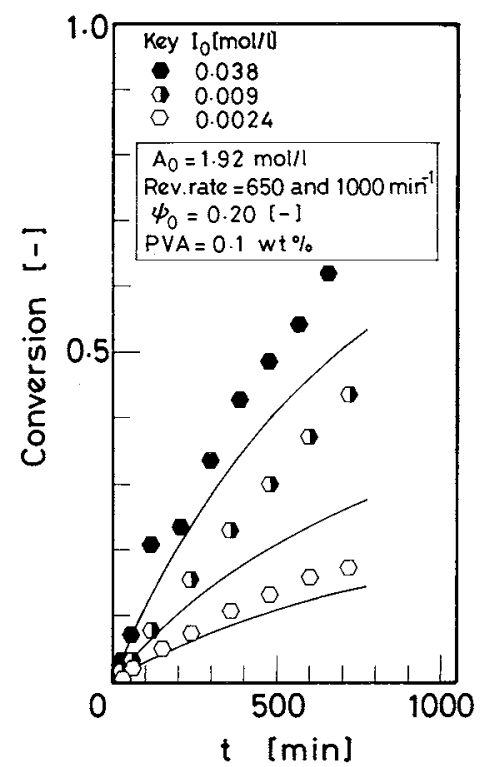

Fig. 12. Effect of initiator concentration on polymerization rate $\left(A_{0}=1.92 \mathrm{~mol} / l\right)$.

of $f$ is assumed, Eq. (6) is inconsistent with the experimental results. At various initial monomer concentrations, except for $A_{0}=5.1 \mathrm{~mol} / l$, the relations between $1 / \bar{P}_{N 0}$ and $I_{0} / R_{P 0}$ are represented in Fig. 14. It is obvious from the figure that the slopes of the lines are different from each other, being steeper with

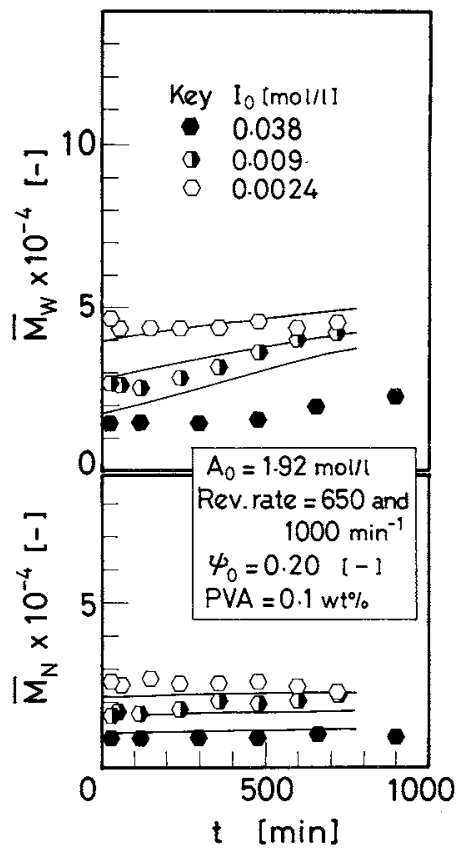

Fig. 13. Effect of initiator concentration on average molecular weight $\left(A_{0}=1.92 \mathrm{~mol} / \mathrm{l}\right)$.

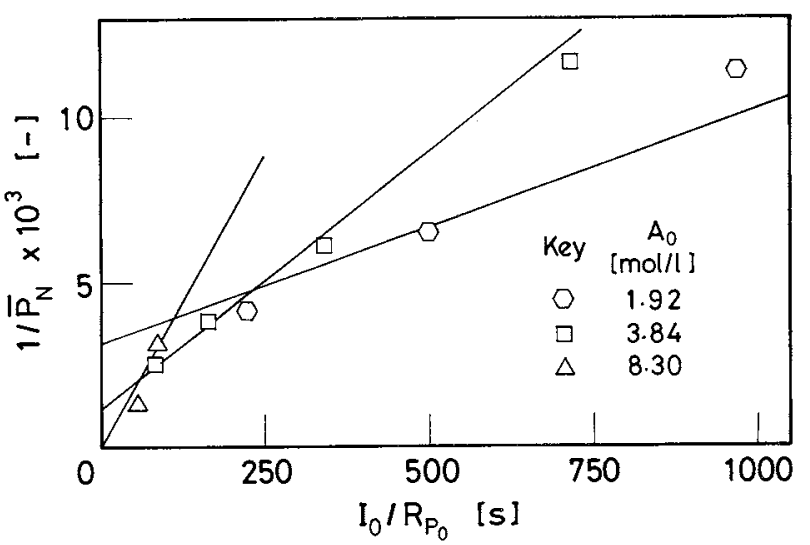

Fig. 14. Relations between $1 / \bar{P}_{N O}$ and $I_{0} / R_{P 0}$.

Table 3. $f$ values from Figs. 11 and 14

\begin{tabular}{ccl}
\hline $\begin{array}{c}f \\
{[-]}\end{array}$ & $\begin{array}{c}A_{0} \\
{[\mathrm{~mol} / l]}\end{array}$ & $\begin{array}{c}\text { SWF } \\
{[-]}\end{array}$ \\
\hline 0.838 & 8.30 & 0 \\
0.545 & 5.3 & 0.32 \\
0.370 & 3.8 & 0.47 \\
0.160 & 1.9 & 0.72 \\
\hline
\end{tabular}

SWF: solvent weight fraction

increasing initial monomer concentration. Calculated $f$ from the slope of each line in Figs. 11 and 14 is listed with each corresponding $A_{0}$ in Table 3 . It seems reasonable from the table to assume that isooctane, a poor solvent for polymer, acts to lower initiator efficiency. Hence, $f$ is plotted against solvent weight fraction (SWF) in Fig. 15. The following empirical 


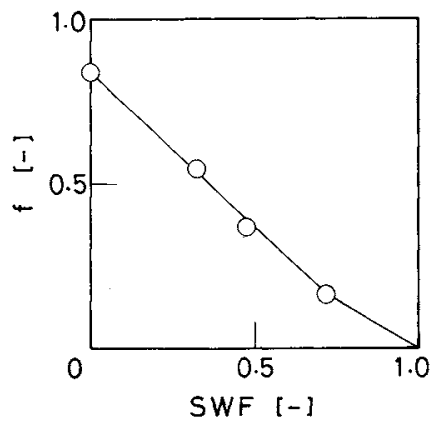

Fig. 15. Relation between $f$ and SWF.

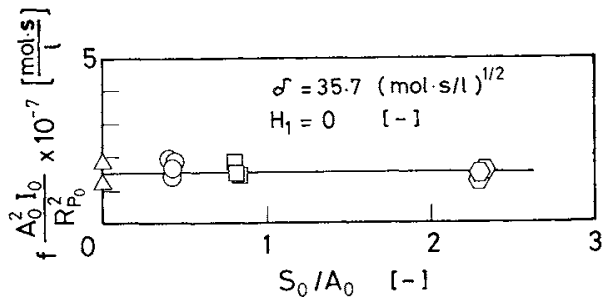

Fig. 16. Relation between $f I_{0} A_{0}^{2} / R_{P 0}^{2}$ and $S_{0} / A_{0}$.

correlation was obtained for initiator efficiency.

$$
\begin{array}{lll}
f=0.838(1-1.129 \mathrm{SWF}) & \text { for } & \mathrm{SWF} \leq 0.72 \\
f=0.560(1-\mathrm{SWF}) & \text { for } & \mathrm{SWF}>0.72
\end{array}
$$

From the intersections at the ordinate of the straight lines in Figs. 11 and 14, $T_{f s}$ was determined using a $T_{f A}$ value from the literature ${ }^{2)}$ of $1.35 \times 10^{-3}$ $[-]$. Here, $T_{f A}=0.843 \times 10^{-4}[-]$.

The following equation, transformed from Eq. (1), is used to obtain $\delta\left(=k_{t}^{1 / 2} / k_{p}\right)$ and $H_{1}$.

$$
\frac{f I_{0} A_{0}^{2}}{R_{p_{0}}^{2}}=\frac{\delta}{2 k_{d}}\left(1+2 T_{f S} H_{1} \frac{S_{0}}{A_{0}}\right)
$$

Relations of $f I_{0} A_{0}^{2} / R_{P 0}^{2}$ vs. $S_{0} / A_{0}$ are plotted over a wide range of $A_{0}$ from 8.3 to $1.9 \mathrm{~mol} / /$ in Fig. 16. The line obtained is almost parallel with the abscissa. Hence $H_{1}=0 . \quad \delta=35.7 \quad(\mathrm{~mol} \cdot \mathrm{s} / l)^{1 / 2}$ from the intersection.

3.1.3 Effect of monomer concentration on the polymerization Effect of monomer concentration on the rate of polymerization and the average molecular weight, respectively, is shown in Figs. 17 and 18. With decreasing initial monomer concentration, monomer conversion rate decreases in the lowconversion region. However, the differences in $x$ vs. $t$ relation among data covering a wide range of $A$ are small in the high-conversion region. Therefore, $S$ shaped relations between $x$ and $t$ are observed at low initial monomer concentration. Such an $x$ vs. $t$ relation is characteristic in common slurry polymerization.

With decreasing initial monomer concentration, the average molecular weight of produced polymer also decreases. At low initial monomer concentrations, the

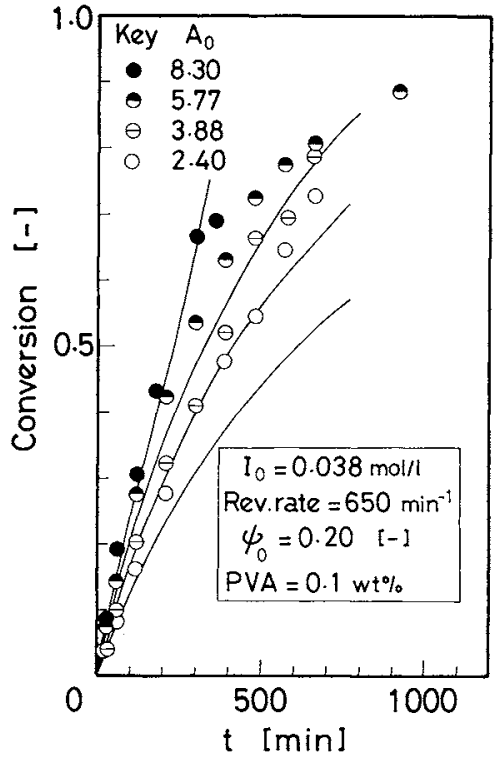

Fig. 17. Effect of monomer concentration on polymerization rate.

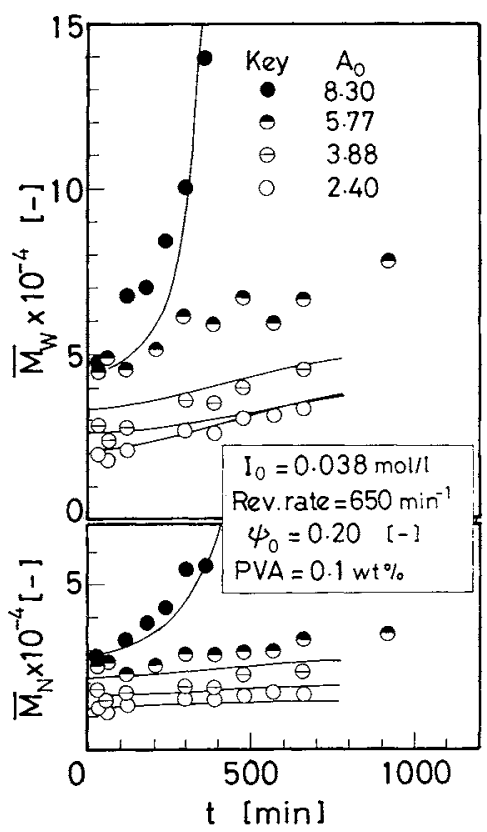

Fig. 18. Effect of monomer concentration on average molecular weight.

following are the characteristics found in this polymerization system: 1) polymers obtained are confirmed to have much higher average molecular weight than those in homogeneous solution polymerization, and 2) $\bar{M}$ increases with reaction time or is almost independent of reaction time, which is contrary to homogeneous solution polymerization.

\subsection{Kinetic equations from a two-phase separation model ${ }^{* 2}$}

This polymerization apparently proceeds in a

*2 No effect of water on the polymerization kinetics is considered. Hence, the organic phase alone is assumed to form the system. 
liquid-solid system, precipitating polymer particles. In fact, solid particles were observed in the samples from the reactor. However, such phenomenon occurs due to the abrupt decrease in temperature from $70^{\circ} \mathrm{C}$ to room temperature. In an actual polymerization system, a two-phase separation may occur at the point where polymer produced exceeds the solubility. One phase is considered to be a solvent-poor phase of monomer and isooctane being free from polymer, and the other is a polymer-rich phase involving monomer, isooctane and polymer.

As long as the system is homogeneous, the same rate equations as shown in the previous papers ${ }^{3-5}$ ) can be used to predict the kinetics. However, the following rate equations based on a two-phase separation model should be used to analyze reactor performance after separation occurs in the system.

Two-phase separation model In this model, the system is assumed to separate into two homogeneous phases, that is, a solvent-poor phase containing no polymer and a polymer-rich phase. Concentration equilibria between the two phases of monomer, solvent and initiator would be reached. Gel effect would be predominant only in the polymer-rich phase.

Assigning 1 and 2 to the solvent-poor phase and the polymer-rich phase respectively, the following equilibrium ratios of monomer, solvent and initiator are defined:

$$
\begin{aligned}
& Q_{A}=A_{2} / A_{1} \\
& Q_{S}=S_{2} / S_{1} \\
& Q_{I}=I_{2} / I_{1}
\end{aligned}
$$

$Q_{A}, Q_{S}$ and $Q_{I}$ can be written as functions of monomer concentration alone in the solvent-poor phase $\left(=A_{1}\right)$.

The volume fraction of the solvent-poor phase $\left(=\chi_{v}\right)$ is a solution of the following equation:

$$
\frac{a_{0}(1-x) / \phi}{\chi_{v}\left(1-Q_{A}\right)+Q_{A}}+\frac{b_{0} / \phi}{\chi_{v}\left(1-Q_{S}\right)+Q_{S}}=1
$$

where $\phi$ is the shrinking coefficient of the system, and $a$ and $b$ are, respectively, initial volume fractions of monomer and solvent $\left(a_{0}+b_{0}=1\right)$.

$Q_{A}$ and $Q_{S}$ are determined from $A_{1}$, which satisfies the following equation:

$$
A_{1}=\frac{A_{0}(1-x) / \phi}{\chi_{v}\left(1-Q_{A}\right)+Q_{A}}
$$

Polymerization rates in the solvent-poor phase, in the polymer-rich phase and in the overall phase (respectively, $R_{P 1}, R_{P 2}$ and $R_{P}$ ) are written as follows:

$$
R_{p 1}=\frac{k_{p}}{k_{t}^{1 / 2}} \frac{\left(2 k_{d} f\right)^{1 / 2} A_{1} I_{1}^{1 / 2}}{\left(1+2 H_{1} T_{f S} S_{1} / A_{1}\right)^{1 / 2}}
$$

$$
\begin{aligned}
R_{p 2} & =\frac{\gamma}{\varepsilon^{1 / 2}} \frac{k_{p}}{k_{t}^{1 / 2}} \frac{\left(2 k_{d} f \varepsilon\right)^{1 / 2} A_{2} I_{2}^{1 / 2}}{\left(1+2 H_{1} T_{f S} S_{2} / A_{2}\right)^{1 / 2}} \\
& =\gamma \frac{k_{p}}{k_{t}^{1 / 2}} \frac{\left(2 k_{d} f\right) A_{2} I_{2}^{1 / 2}}{\left(1+2 H_{1} T_{f S} S_{2} / A_{2}\right)^{1 / 2}}
\end{aligned}
$$

$$
\begin{aligned}
R_{p}= & \chi_{v} R_{p 1}+\left(1-\chi_{v}\right) R_{p 2} \\
= & \frac{k_{p}\left(2 k_{d} f\right)^{1 / 2} A_{0}(1-x) I_{0}^{1 / 2} \exp \left(-k_{d} t / 2\right) / \phi^{3 / 2}}{k_{t}^{1 / 2}\left\{\chi_{v}\left(1-Q_{A}\right)+Q_{A}\right\}\left\{\chi_{v}\left(1-Q_{I}\right)+Q_{I}\right\}^{1 / 2}} \\
& \times\left\{\frac{\chi_{v}}{\left(1+2 H_{1} Z\right)^{1 / 2}}+\gamma \frac{\left(1-\chi_{v}\right) Q_{A} Q_{I}^{1 / 2}}{\left(1+2 H_{1} Z Q_{S} / Q_{A}\right)^{1 / 2}}\right\}
\end{aligned}
$$

where

$$
\begin{aligned}
& S_{1}=\frac{S_{0} / \phi}{\chi_{v}\left(1-Q_{S}\right)+Q_{S}} \\
& I_{1}=\frac{I_{0} \exp \left(-k_{d} t\right) / \phi}{\chi_{v}\left(1-Q_{I}\right)+Q_{I}} \\
& Z=T_{f S} \frac{S_{0}}{A_{0}(1-x)} \frac{\chi_{v}\left(1-Q_{A}\right)+Q_{A}}{\chi_{v}\left(1-Q_{S}\right)+Q_{S}}
\end{aligned}
$$

$\gamma$ and $\varepsilon$ are, respectively, correction factors for overall polymerization rate and initiator efficiency in the gel effect region. They are described approximately as functions of polymer weight fraction. ${ }^{3,4)}$

Overall production rate of $M_{r}$ is written by the following equation:

$$
\begin{aligned}
\rho \frac{d\left(M_{r} / \rho\right)}{d t}= & \chi_{v}\left\{\rho_{1} \frac{d\left(M_{r 1} / \rho_{1}\right)}{d t}\right\}+\left(1-\chi_{v}\right)\left\{\rho_{2} \frac{d\left(M_{r 2} / \rho_{2}\right)}{d t}\right\} \\
= & \chi_{v} \zeta_{1}^{r-1}\left(1-\zeta_{1}\right)\left[R_{p 1}\left(Z+T_{f A}\right)\right. \\
& \left.+\left\{\frac{(r-1)\left(1-\zeta_{1}\right)}{2}+H_{1} Z\right\} \frac{R_{i 1}}{1+2 H_{1} Z}\right] \\
& +\left(1-\chi_{v}\right) \zeta_{2}^{r-1}\left(1-\zeta_{2}\right)\left[R_{p 2}\left(Z \frac{Q_{S}}{Q_{A}}+T_{f A}\right)\right. \\
& \left.+\left\{\frac{(r-1)\left(1-\zeta_{2}\right)}{2}+H_{1} Z\right\} \frac{R_{i 2}}{1+2 H_{1} Z Q_{S} / Q_{A}}\right]
\end{aligned}
$$

where

$$
\begin{aligned}
& R_{i 1}=2 k_{d} f I_{1}, \quad R_{i 2}=2 k_{d} f \varepsilon I_{2} \\
& \zeta_{1}=1-\left(Z+T_{f A}+\frac{R_{i 1}}{R_{p 1}} \frac{1+H_{1} Z}{1+2 H_{1} Z}\right) \\
& \zeta_{2}=1-\left(Z \frac{Q_{S}}{Q_{A}}+T_{f A}+\frac{R_{i 2}}{R_{p 2}} \frac{1+H_{1} Z Q_{S} / Q_{A}}{1+2 H_{1} Z Q_{S} / Q_{A}}\right)
\end{aligned}
$$

Hence, instantaneous number average degree of polymerization is written by the following equation:

$$
\bar{P}_{N, \text { inst }}=\frac{R_{p}}{\sum_{r=1}^{\infty}\left\{\rho d\left(M_{r} / \rho\right) / d t\right\}}
$$




$$
\begin{aligned}
= & R_{p} /\left[\chi_{v}\left\{R_{p 1}\left(Z+T_{f A}\right)+R_{i 1} / 2\right\}\right. \\
& \left.+\left(1-\chi_{v}\right)\left\{R_{p 2}\left(Z Q_{S} / Q_{A}+T_{f A}\right)+R_{i 2} / 2\right\}\right]
\end{aligned}
$$

Number and weight average degrees of polymerization are calculated from the following equations:

$$
\begin{aligned}
& \bar{P}_{N}=\int_{0}^{t} R_{p} d t / \int_{0}^{t} \sum_{r=1}^{\infty} \rho \frac{d\left(M_{r} / \rho\right)}{d t} d t \\
& \bar{P}_{W}=\int_{0}^{t} \sum_{r=1}^{\infty} r^{2} \rho \frac{d\left(M_{r} / \rho\right)}{d t} d t / \int_{0}^{t} R_{p} d t
\end{aligned}
$$

However, the proposed two-phase separation model might overlook a precipitation of microparticles at the initial stage of the heterogeneous polymerization.

\subsection{Determination of kinetic parameters}

3.3.1 Homogeneous polymerization Kinetic parameters in the absence of gel effect were obtained in section 3.1, and are listed in Table 4. Correction factors $\gamma$ and $\varepsilon$, respectively, for overall polymerization rate and initiator efficiency under gel effect were determined by the established method, ${ }^{3.4 t}$ and are shown in Figs. 19 and 20 as functions of polymer weight fraction (PWF).

3.3.2 Determination of kinetic parameters for two separated phases Kinetic parameters in the solventpoor phase are the same as those of homogeneous polymerization in the absence of gel effect.

$Q_{A}, Q_{S}$ and $Q_{I} \quad$ To estimate $Q_{A}$ and $Q_{S}$, toluene was used instead of styrene. Equilibrium experiments with the toluene-isooctane-polystyrene system were carried out at $70^{\circ} \mathrm{C}$. After a small amount of AIBN was charged in the system, $Q_{I}$ was obtained from AIBN concentrations in both phases. Observed equilibrium compositions are shown on a triangular diagram in Fig. 21. Almost no effect of molecular weight of polymer samples $\left(\bar{M}_{w}=3.19 \times 10^{4}\right.$ and $\left.17.4 \times 10^{4}\right)$ was detected, as shown in Fig. 21. The lower area FPG represents the region of immiscibility and the borderline curve FPG represents the solubility limits of the system. The bold line PH in the immiscible region is the conjugate line. As expected, almost no polymer content is recognized in the solvent-poor phase.

The relation between each $Q$-value and $A$ is shown in Fig. 22.

$\gamma$ and $\varepsilon$ Since the gel effect is predominant only in the polymer-rich phase, the polymerization rate in the polymer-rich phase must be regarded as being equal to the overall polymerization rate to obtain $\gamma$ and $\varepsilon$ from the following calculations.

Under the conditions of small contribution of the solvent-poor phase to the overall reaction, $\gamma$ can be

\begin{tabular}{|c|}
\hline $\begin{array}{ll}\delta \quad=35.7 & {[\mathrm{~mol} \cdot \mathrm{s} / /]^{1 / 2}} \\
T_{f A}{ }^{2)}=0.843 \times 10^{-4} & {[-]} \\
T_{f S} 1.35 \times 10^{-3} & {[-]} \\
k_{d}^{2)}=4.25 \times 10^{-5} \quad[\mathrm{~s}] & \\
H_{1}=0 & {[-]} \\
f=0.838(1-1.129 \mathrm{SWF}) & \text { for } \quad \mathrm{SWF} \leq 0.72 \\
f=0.560(1-\mathrm{SWF}) & \text { for } \quad \mathrm{SWF}>0.72 \\
& \end{array}$ \\
\hline
\end{tabular}
determined from the following equation to be correlated with PWF.
Table 4. Kinetic parameters

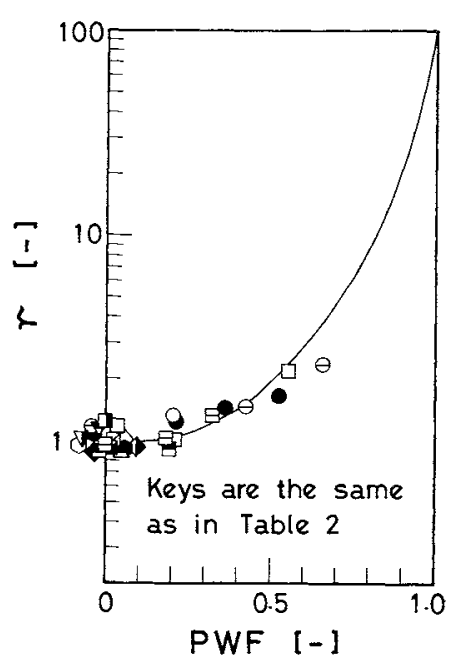

Fig. 19. $\gamma$ versus PWF (homogeneous polymerization).

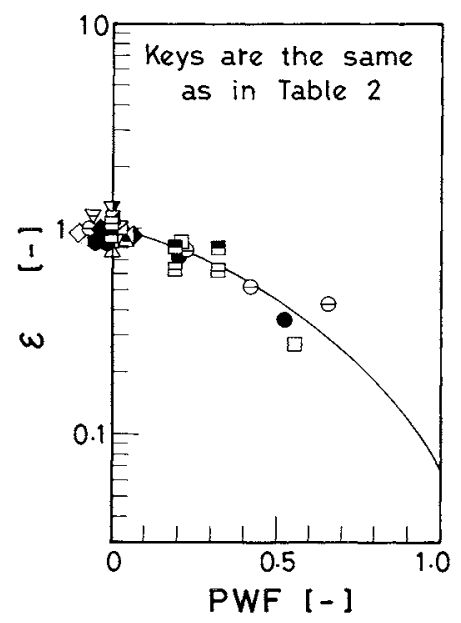

Fig. 20. $\varepsilon$ versus PWF (homogeneous polymerization).

$$
\gamma=\frac{R_{p, \mathrm{ob}}-\chi_{v} R_{p 1, \mathrm{cal}}}{\left(1-\chi_{v}\right) \frac{k_{p}}{k_{t}^{1 / 2}} \frac{\left(2 k_{d} f\right)^{1 / 2} A_{2} I_{2}^{1 / 2}}{\left(1+2 H_{1} T_{f S} S_{2} / A_{2}\right)^{1 / 2}}}
$$

In the condition where $\chi_{v} R_{P 1, \text { cal }} / R_{P, \text { ob }}$ is almost zero, polymer obtained is considered to be the polymer from the polymer-rich phase. Instantaneous number average degree of polymerization in this region is obtained from the following equation:

$$
\bar{P}_{N, \text { inst }}=\frac{\bar{P}_{N}}{1-\frac{\left(d \bar{P}_{N} / d t\right) A_{0}(1-x) / \phi}{\bar{P}_{N} \cdot R_{p}}}
$$




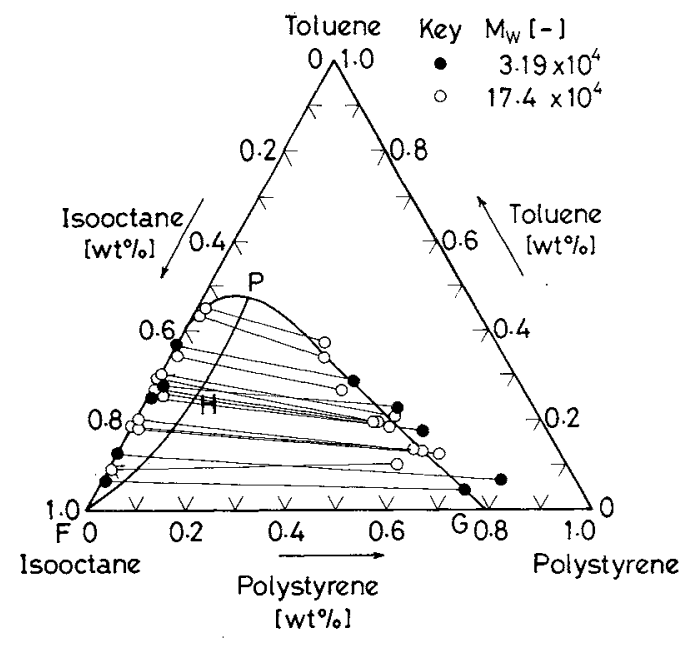

Fig. 21. Observed equilibrium composition.

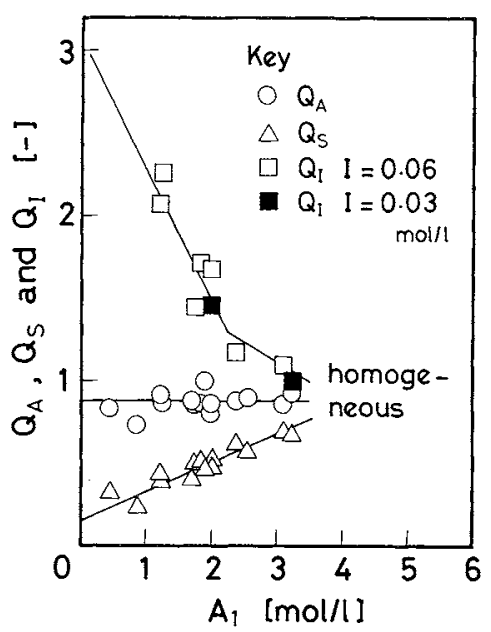

Fig. 22. Relation between each $Q$-value and $A$.

$\varepsilon$ can be determined by the following equation, derived from Eq. (22).

$$
\varepsilon=\frac{R_{p}}{f k_{d} I_{2}}\left[\frac{1}{\bar{P}_{N, \text { inst }}}-\left(Z \frac{Q_{S}}{Q_{A}}+T_{f A}\right)\right]
$$

$\gamma$ and $\varepsilon$ are, respectively, shown as functions of PWF in Figs. 23 and 24. It is evident in the range of the experimental conditions that $\gamma$ and $\varepsilon$ can be described as a function of PWF alone. The same solid lines as in Figs. 23 and 24 are, respectively, drawn in Figs. 19 and 20 to show the same gel effect in both cases.

3.3.3 Calculation of conversion and average molecular weight Using Eqs. (17), (23) and (24) with the empirical kinetic parameters in Table 4 and Figs. 23 and 24, time dependencies of conversion and molecular weight were calculated for each experimental condition. Calculated results are shown as solid lines in Figs. 2-9, Figs. 12 and 13 and Figs. 17 and 18.

Applicability of the two-phase separation model to this polymerization has been proved by close agreement between observed and calculated values for both

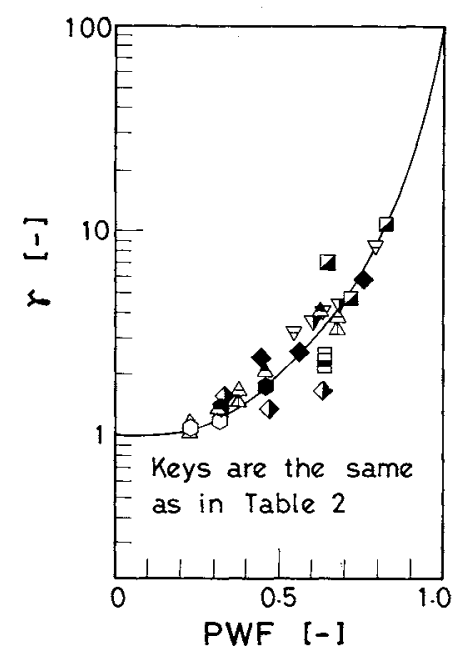

Fig. 23. $\gamma$ versus PWF (heterogeneous polymerization).

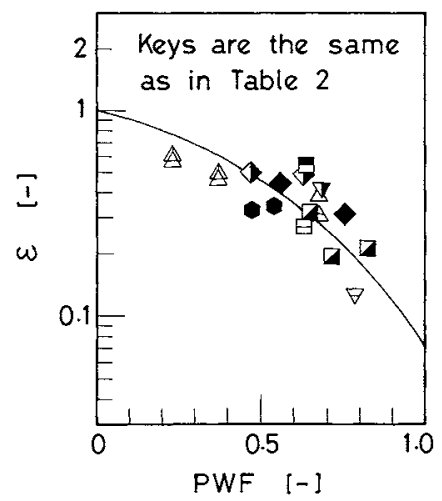

Fig. 24. $\varepsilon$ versus PWF (heterogeneous polymerization).

the conversion and the average molecular weight of polymer produced.

\section{Conclusion}

Kinetic research was carried out to clarify the characteristics of styrene slurry polymerization in suspended isooctane droplets. The following results were obtained.

1) Important operating factors such as revolution rate, existence of PVA and volume fraction of dispersion phase have no effect on the kinetics of the polymerization in the same manner as the common suspension polymerization in the range of experimental conditions.

2) A common homogeneous solution polymerization occurs when the organic phase is homogeneous. However, considerable auto-accelerating effects on the time-dependencies of conversion and average molecular weight are found on and after the appearance of a polymer-rich phase.

3) A two-phase separation model is proposed, in which the separation is described as a changing process from a homogeneous phase to two phases; one involving monomer and solvent in the absence of gel effect, and the other involving monomer solvent 
and polymer in the presence of gel effect. Furthermore, equilibria of monomer, solvent and initiator between the phases are assumed to be reached in the model.

4) Empirical kinetic parameters in the rate equations based on the two-phase separation model were determined to describe the relations of conversion and average molecular weight with reaction time.

\section{Nomenclature}

$=$ initial volume fraction of monomer

[mol/l]

$=$ initial volume fraction of solvent

$f \quad=$ initiator efficiency

$H_{1} \quad=\left(k_{t s} / k_{t}\right) /\left(k_{i s} / k_{p}\right)$ kinetic parameter

$I \quad=$ initiator or initiator concentration

$I^{*} \quad=$ initiator radical

$k \quad=$ reaction rate constants for $k_{d}=$ initiator decomposition $k_{f \boldsymbol{A}}, k_{f S}=$ chain transfers to monomer and solvent, respectively

$k_{i}=$ initiation

$k_{i s}=$ re-initiation by solvent radical

$k_{p}=$ propagation

$k_{t}=$ termination

$\dot{k}_{t c}, k_{t d}=$ terminations by recombination and disproportionation, respectively

$k_{t S}=$ termination by solvent

$\bar{M}_{N} \quad$ = number average molecular weight

$\bar{M}_{W} \quad=$ weight average molecular weight

$M_{r} \quad=$ dead polymer consisting of $r$-monomer units or its concentration

$P \quad=$ active polymer

$\bar{P}_{N} \quad=$ number average degree of polymerization

$\bar{P}_{W} \quad=$ weight average degree of polymerization

PVA $=$ polyvinyl alcohol or its concentration in water phase

$Q_{A}, Q_{I}, Q_{S}=$ equilibrium ratios defined in Eqs. (10), (11) and (12), respectively

$R_{i} \quad=$ initiation rate

$R_{p} \quad=$ polymerization rate

$r \quad=$ number of monomer units

\begin{tabular}{|c|c|c|c|}
\hline$S$ & $=$ & solvent or solvent concentration & {$[\mathrm{mol} / \mathrm{l}]$} \\
\hline$T_{f A}, T_{f S}$ & $=$ & $\begin{array}{l}\text { chain transfer constants to monomer or } \\
\text { solvent, respectively }\end{array}$ & {$[-]$} \\
\hline$t$ & $=$ & reaction time & \\
\hline$x$ & $=$ & monomer conversion & {$[-]$} \\
\hline$Z$ & $=$ & kinetic parameter defined in Eq. (20) & \\
\hline$\gamma$ & $=$ & $\begin{array}{l}\text { correction factor for overall reaction rate } \\
\text { under gel effect }\end{array}$ & {$[--]$} \\
\hline$\delta$ & $=$ & $k_{t}^{1 / 2} / k_{p}$, kinetic constant $\quad[(\mathrm{mol}$ & $\left.\cdot \mathrm{s} / l)^{\mathrm{L} / 2}\right]$ \\
\hline$\varepsilon$ & $=$ & $\begin{array}{l}\text { correction factor for initiator efficiency } \\
\text { under gel effect }\end{array}$ & {$[-]$} \\
\hline$\zeta$ & $=$ & $\begin{array}{l}\text { probability factor for calculation of degree } \\
\text { of polymerization }\end{array}$ & {$[-]$} \\
\hline$\rho$ & $=$ & $\rho_{0} / \phi$, density of organic phase & {$[\mathrm{kg} / l]$} \\
\hline$\rho_{A}, \rho_{P}$ & $=$ & densities of monomer and polymer, respect & $\begin{array}{l}\text { tively } \\
\qquad[\mathrm{kg} /\end{array}$ \\
\hline$\phi$ & $=$ & $\begin{array}{l}1-0.1041 A_{0} \times\left(1-\rho_{A} / \rho_{P}\right) / \rho_{A}, \text { shrinking } \\
\text { coefficient of organic phase }\end{array}$ & {$[-]$} \\
\hline$\chi_{v}$ & $=$ & $\begin{array}{l}\text { volume fraction of poor-solvent phase in } \\
\text { organic phase }\end{array}$ & \\
\hline 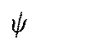 & $=$ & volume fraction of dispersion phase & {$[-]$} \\
\hline
\end{tabular}

$\langle$ Subscripts〉

$\begin{array}{ll}0 & =\text { initial state } \\ 1 & =\text { poor-solvent phase } \\ 2 & =\text { polymer-rich phase } \\ \text { cal } & =\text { calculated } \\ \text { inst } & =\text { instantaneous } \\ \text { ob } & =\text { observed }\end{array}$

\section{Literature Cited}

1) Ahmed, H. A. and A. E. Hamielec: J. Appl. Polym. Sci., 16, 783 (1972).

2) Brandrup, J. and E. H. Immergut, ed.: "Polymer Handbook," John Wiley \& Sons (1975).

3) Harada, M., T. Yamada, K. Tanaka, W. Eguchi and S. Nagata: Kagaku Kōgaku, 29, 301 (1965).

[wt $\%$ 4) Hatate, Y., T. Hano, T. Miyata, F. Nakashio and W. Sakai: Kagaku Kögaku, 35, 903 (1971).

5) Hatate, Y. and F. Nakashio: Kagaku Kögaku, 37, 171 (1973).

6) Hatate, Y., A. Ikari, F. Nakashio and K. Kondo: J. Chem. Eng. Japan, 17, 339 (1984).

7) Saeki, K.: "Polymer Seizo Process," Kogyo Chosa Kai (1971). 